\title{
Pathogenesis of bloodstream infection in children with blood cancer
}

\author{
HUA LV ${ }^{1}$ and BOTAO NING ${ }^{2}$ \\ Departments of ${ }^{1}$ Infection Control and ${ }^{2}$ Pediatric ICU, Children's Hospital, School of \\ Medicine, Zhejiang University, Hangzhou, Zhejiang 310003, P.R. China
}

Received July 5, 2012; Accepted September 20, 2012

DOI: $10.3892 /$ etm.2012.738

\begin{abstract}
The aim of the present study was to characterize the distribution and antibiotic resistance of pathogens isolated from patients with bloodstream infections (BSIs) in the Hematology and Oncology department of the Affiliated Children's Hospital of Zhejiang University Medical School (Hangzhou, China), between January and December 2010 and to provide early and appropriate support for the clinical administration of antibiotics. Out of 1,500 inpatients, 161 children who were diagnosed with BSI based on the national diagnostic criteria were retrospectively analyzed. Neutropenia was defined as an absolute neutrophil count (ANC) in the peripheral blood of less than $0.5 \times 10^{9}$ cells $/ 1$. A microbiologically documented infection (MDI) was defined as when the causative pathogen was isolated from the blood. Drug susceptibility tests were performed using a VITEK-60 AutoMicrobic System and the Kirby-Bauer disk diffusion method. The data were analyzed using STATA software (version 9.0) and a two-sided P-value of $\leq 0.05$ was considered to indicate a statistically significant difference. A total of 79 strains were isolated from the blood specimens. The incidence of BSI was $10.73 \%$ (161/1,500). Gram-positive cocci, Gram-negative bacilli and fungi accounted for 55.70, 43.04 and $1.27 \%$ of the BSIs, respectively. Staphylococcus epidermidis $(20.25 \%)$, Escherichia coli $(15.19 \%)$ and Klebsiella pneumoniae (15.19\%) were frequently identified isolates. The staphylococci were susceptible to vancomycin and linezolid, while Escherichia coli and Klebsiella pneumoniae were sensitive to cefoperazone/sulbactam, imipenem and meropenem. In conclusion, Gram-positive bacteria are slightly more prevalent than Gram-negative bacteria in BSI and the selection of antibiotics according to the susceptibility test results is superior to empirical treatment. It is essential to administer antimicrobial agents early and appropriately to treat child blood cancer patients with BSI.
\end{abstract}

Correspondence to: Dr Botao Ning, Department of Pediatric ICU, Children's Hospital, School of Medicine, Zhejiang University, 57 Zhugang Lane, Hangzhou, Zhejiang 310003, P.R. China

E-mail: ningbotao@126.com

Key words: bloodstream infection, pathogen, drug resistance

\section{Introduction}

Sepsis and bacteremia are referred to as bloodstream infection (BSI) (1). Children with blood cancer often suffer from neutropenia, immune suppression and damage to the skin, oral cavity and mucous membrane of the digestive tract due to cancer, chemotherapy, radiotherapy and the administration of immunosuppressants. This allows invasive and conditional bacteria to invade the bloodstream through the mucous membrane barrier (2). In order to elucidate the pathogenic distribution of BSI and provide strategies for its prevention and treatment, an investigation of BSI in patients with blood cancer was performed at the Affiliated Children's Hospital of Zhejiang University Medical School (Hangzhou, China) between January and December 2010.

\section{Patients and methods}

Definitions. Neutropenia was defined as an absolute neutrophil count (ANC) in the peripheral blood of $<0.5 \times 10^{9}$ cells $/ 1(3,4)$. Febrile patients were defined as those yielding 1 axillary temperature measurement of $\geq 38.5^{\circ} \mathrm{C}$ or 2 measurements of $38-38.4^{\circ} \mathrm{C}$ within a $4-h$ interval (4). BSI was diagnosed according to the infection diagnostic standards published by the Medical Administration Department of the Chinese Health Ministry in 2001 (5). A clinical diagnosis was defined as when temperature was $>38^{\circ} \mathrm{C}$ or $<36^{\circ} \mathrm{C}$ and occurred with one of the following conditions: i) invasion or migration lesions; ii) systemic poisoning symptoms without an apparent focal infection; iii) unexplained skin rash, petechia, hepatosplenomegaly or increased neutrophil count with a left shift; iv) systolic blood pressure $<12 \mathrm{kPa}(90 \mathrm{mmHg})$ or a blood pressure decrease of $>5.3 \mathrm{kPa}(40 \mathrm{mmHg}$ ) compared with the original systolic pressure. An etiological diagnosis was defined according to the clinical diagnosis and the occurrence of one of the following events: i) a pathogenic microorganism was cultured from the blood; ii) a pathogen antigen was detected in the blood.

Patients. At the Affiliated Children's Hospital of Zhejiang University Medical School between January and December 2010, 1,500 new patients were admitted to the hematology department. Of these, 161 patients ( 93 males and 68 females) with a median age of 7 years (range, 1 month to 17 years) developed BSI. The basic diseases of the BSI patients 
consisted of 112 cases of acute lymphocytic leukemia, 34 of acute myelogenous leukemia, 10 of lymphoma, 4 of hemophagocytic syndrome and 1 of Langerhans cell histiocytosis. The etiologically diagnosed patients with positive blood culture were categorized as the microbiologically documented infection (MDI) group and the clinically diagnosed patients with negative blood culture were the non-MDI group $(6,7)$. The study was approved by the ethics committee of the Children's Hospital affiliated to the School of Medicine of Zhejiang University, and written, informed consent was obtained from the patients' parents or legal guardians in all cases.

Instruments and reagents. Bacteria were identified using a VITEK-60 AutoMicrobic System purchased from the Marcel Mérieux Company, Lyon, France. Susceptibility testing paper and susceptibility medium (MH medium) were purchased from the Oxiod Ltd. (Basingstoke, UK) and Marcel Mérieux, respectively.

Antibiotic susceptibility test. A total of 161 blood samples were cultured and routine drug susceptibility tests were performed using the VITEK-60 AutoMicrobic System and the Kirby-Bauer disk diffusion method. The extended-spectrum $\beta$-lactamase (ESBL) strains were detected using a double disk test. The drug resistance criteria followed the Clinical Laboratory Standards Institute (CLSI) guidelines set in 2008 (8). If more than 2 consecutive results were the same in one patient, the results of the blood culture were recorded once; if the results differed, each result of the blood culture was recorded.

Statistical analysis. The data were analyzed using STATA software, version 9.0 (StataCorp LP, College Station, TX, USA). A two-sided P-value of $\leq 0.05$ was considered to indicate a statistically significant difference.

\section{Results}

Incidence of BSI and rate of MDI. Out of 1,500 child patients, 161 were diagnosed as having BSI. The incidence was $10.73 \%$ $(161 / 1,500)$ and 79 of the blood cultures were positive so the positive rate of the blood culture was $49.07 \%$ (79/161).

Distribution of pathogens. A total 79 strains were detected in 161 patients. Gram-positive bacteria, Gram-negative bacteria and fungi accounted for 55.70 (44/79), 43.04 (34/79) and 1.27\% (1/79) of the BSIs, respectively. The most common pathogens were Staphylococcus epidermidis, Escherichia coli and Klebsiella pneumoniae which accounted for 20.25, 15.19 and $15.19 \%$, respectively. The distribution of pathogens is shown in Table I.

Drug resistance results of common pathogens. A total of $27 \beta$-lactamase-positive strains were detected from the 31 identified staphylococci, accounting for $87.1 \%$ (27/31). A total of 16 Staphylococcus epidermidis strains exhibited 100\% resistance to oxacillin, $72.73 \%$ of the 11 strains of other coagulase-negative staphylococci (CoNS) were resistant to oxacillin and the 4 strains of Staphylococcus aureus were not resistant to oxacillin. A total of 9 ESBL-producing strains were detected from 12 strains of Escherichia coli (75\%) and
Table I. Distribution of BSI pathogens.

\begin{tabular}{lcc}
\hline Pathogens & $\begin{array}{c}\text { Number of } \\
\text { strains }\end{array}$ & $\begin{array}{c}\text { Proportion } \\
(\%)\end{array}$ \\
\hline Gram-positive bacteria & 44 & 55.70 \\
Staphylococcus epidermidis & 16 & 20.25 \\
Other CoNS & 11 & 13.92 \\
Streptococcus & 6 & 7.59 \\
Staphylococcus aureus & 4 & 5.06 \\
Enterococcus & 4 & 5.06 \\
Micrococcus & 2 & 2.53 \\
Bacillus & 1 & 1.27 \\
Gram-negative bacteria & 34 & 43.04 \\
Escherichia coli & 12 & 15.19 \\
Klebsiella pneumoniae & 12 & 15.19 \\
Pseudomonas aeruginosa & 5 & 6.33 \\
Sphingomonas paucimobilis & 2 & 2.53 \\
Aeromonas hydrophila & 2 & 2.53 \\
Salmonella thompsoni & 1 & 1.27 \\
Fungus & 1 & 1.27 \\
Candida albicans & 1 & 1.27 \\
Total & 79 & 100.00
\end{tabular}

BSI, blood stream infection; CoNS, coagulase-negative staphylococcus

5 ESBL-producing strains were detected from 12 strains of Klebsiella pneumoniae (41.7\%). The staphylococcus and Gram-negative bacteria resistance rates are shown in Tables II and III, respectively.

Treatment and outcome. All the 161 BSI patients were treated with antimicrobial agents, which included $\beta$-lactams, carbapenems, glycopeptides, nitroimidazoles and anti-fungals. A total of 30 patients (18.63\%) received single drugs, 63 patients (39.13\%) received 2 drugs and 68 patients (42.24\%) received 3 or more drugs. The 5 most commonly used antimicrobial agents were vancomycin, imipenem, cefoperazone/sulbactam, piperacillin/tazobactam and meropenem. The mean treatment times were was $10.77 \pm 4.88$ days for the non-MDI group and $8.27 \pm 2.85$ days for the MDI group ( $\mathrm{t}=3.81, \mathrm{P}=0.0002)$. Granulocyte colony-stimulating factor (G-CSF) and venous injections of immunoglobulin were administered to the patients with neutropenia at the same time as the antimicrobial therapy. With regard to the fungal infection, oral fluconazole was administered for 3 days while ANC was $<1.0 \times 10^{9}$ cells $/ 1$. Of the 161 patients, 156 were successfully cured and 5 BSI patients succumbed to uncontrolled systemic infection. One of these patients was infected with Pseudomonas aeruginosa, 2 were infected with Klebsiella pneumoniae and 2 were negative in the blood culture. The mortality rate was $3.1 \%(5 / 161)$.

\section{Discussion}

The blood cancer patients were susceptible to infection complications, particularly bacterial sepsis, as well as low 
Table II. Drug resistance pattern of staphylococci isolated from the blood of patients with BSI.

\begin{tabular}{lrrr}
\hline & & Drug resistance rate $(\%)$ \\
\cline { 2 - 4 } Drugs & Staphylococcus epidermidis $(\mathrm{n}=16)$ & Other CoNS $(\mathrm{n}=11)$ & Staphylococcus aureus $(\mathrm{n}=4)$ \\
\hline Clindamycin & 50.00 & 45.45 & 50.00 \\
Linezolid & 0.00 & 0.00 & 0.00 \\
Ampicillin/sulbactam & 100.00 & 72.73 & 0.00 \\
Gentamicin & 37.50 & 27.27 & 25.00 \\
Oxacillin & 100.00 & 72.73 & 0.00 \\
Rifampicin & 12.50 & 9.09 & 0.00 \\
Sulfamethoxazole & 93.75 & 36.36 & 75.00 \\
Vancomycin & 0.00 & 0.00 & 0.00 \\
Moxifloxacin & 0.00 & 0.00 & 0.00 \\
Erythromycin & 93.75 & 54.55 & 75.00 \\
Furantoin & 0.00 & 0.00 & 0.00 \\
Levofloxacin & 0.00 & 27.27 & 25.00 \\
Penicillin-G & 100.00 & 81.82 & 75.00 \\
Tetracycline & 25.00 & 9.09 & 0.00 \\
\hline
\end{tabular}

BSI, blood stream infection.

Table III. Drug resistance pattern of common Gram-negative bacteria isolated from the blood of patients with BSI.

Drug resistance rate $(\%)$

\begin{tabular}{|c|c|c|c|}
\hline \multirow[b]{2}{*}{ Drugs } & \\
\hline & Escherichia coli $(\mathrm{n}=12)$ & Klebsiella pneumoniae $(\mathrm{n}=12)$ & Pseudomonas aeruginosa $(\mathrm{n}=5)$ \\
\hline Cefoperazone/sulbactam & 0.00 & 0.00 & 100.00 \\
\hline Cephazoline & 83.33 & 50.00 & 100.00 \\
\hline Cefoxitin & 0.00 & 0.00 & 0.00 \\
\hline Amikacin & 8.33 & 0.00 & 0.00 \\
\hline Levofloxacin & 33.33 & 16.67 & 100.00 \\
\hline Cefuroxime & 83.33 & 58.33 & 0.00 \\
\hline Ceftazidime & 75.00 & 50.00 & 100.00 \\
\hline Ampicillin & 100.00 & 83.33 & 0.00 \\
\hline Cefpiramide & 75.00 & 50.00 & 0.00 \\
\hline Gentamicin & 83.33 & 50.00 & 0.00 \\
\hline Imipenem & 0.00 & 0.00 & 0.00 \\
\hline Meropenem & 0.00 & 0.00 & 80.00 \\
\hline Cefotaxime & 75.00 & 41.67 & 0.00 \\
\hline Piperacillin/tazobactam & 0.00 & 25.00 & 0.00 \\
\hline
\end{tabular}

BSI, blood stream infection.

immune function caused by malignant tumors and the impairment of physiological barrier defense function causing topical edema, erosion, necrosis, compression and obstruction. Neutropenia resulting from chemotherapy and/or radiotherapy may be the single most important risk factor responsible for the sepsis (9). The data revealed that the incidence of BSI was $10.73 \%(161 / 1,500)$ versus $5.2-18.2 \%$ as reported previously $(10,11)$. The positive blood culture rate of $49.07 \%$ in BSI patients was lower than that of other studies $(12,13)$. This may be since we did not routinely collect double blood samples from various sites simultaneously.

Our study revealed that the distribution of Gram-positive bacteria, Gram-negative bacteria and fungi was 55.70, 43.04 and $1.27 \%$, respectively. The 3 most common pathogens were Staphylococcus epidermis, Escherichia coli and Klebsiella pneumoniae. Celkan et al (14) reported that $60 \%$ of the pathogens separated from the blood culture of 159 oncological patients were Gram-positive bacteria and the CoNS was the 
dominant. These results were similar to those of the present study. Gram-positive bacterial infection is an increasing trend in cancer patients and the infection rates of CoNS and streptococci have increased the most rapidly (15). This may contribute to the extremely high usage levels of broad spectrum antimicrobial agents for use against Gram-negative bacteria and invasive surgery, such as deep vein catheter implantation. Long-term and extremely high levels of broad spectrum antibiotic use may potentially cause the over-proliferation of Gram-positive bacteria and catheter puncture may result in mucocutaneous injury, making conditional pathogenic infection possible. The most common Gram-positive bacteria (staphylococci) exhibited no or low drug resistance to vancomycin, linezolid and moxifloxacin, while the dominant Gram-negative bacteria had no or low drug resistance to cefoxitin, imipenem and piperacillin/ tazobactam. According to the guidelines of the American Infectious Disease Association (16,17), as well as the supportive treatment, antibiotics were administered early, at adequate levels and in combinations such as imipenem with vancomycin or piperacillin/tazobactam with vancomycin. As soon as the results of the antibiotic susceptibility tests were available, the antibiotics were adjusted accordingly. The mean treatment time of the MDI group was less than that of the non-MDI group and this difference was observed to be statistically significant $(\mathrm{t}=3.81, \mathrm{P}=0.0002)$. This demonstrated that antibiotic selection according to the susceptibility test results was superior to empirical treatment during the whole of the anti-infectious treatment, although the latter was important in the initial period. Of the 5 patients who succumbed to infection, 3 were infected with Gram-negative bacteria and no bacteria were cultured in the remaining 2. Although the mortality rate was low $(3.1 \%, 5 / 161)$, certain patients exhibited negative pathogenic cultures.

Although the fungal infection accounted for $1.27 \%$ (1/161) of the data, various pathogens, such as fungi, viruses and parasites, may infect patients with neutropenia and fungemia may be the prime cause of mortality (18). Mor et al (19) reported that $7.2 \%$ of the 1,047 children hospitalized in a Hematology/Oncology department were diagnosed with a proven invasive fungal infection. The low detection rate of invasive fungal infection in the present study may be due to the difficulty of culturing fungi. The lack of sensitive and specific detection methods possibly contributed to the prophylactic usage of oral fluconazole when the ANC was less than $1.0 \times 10^{9}$ cells $/ 1$.

In conclusion, Gram-positive bacteria are the dominant pathogens in child blood cancer patients with BSI. Antibiotic selection according to the susceptibility test results is superior to empirical treatment. Antibiotic susceptibility tests are extremely important for the drug adjustment. In order to decrease the incidence and improve the outcome of BSI, improved prevention, surveillance of antibiotic resistance, early detection and advanced therapeutic strategies should be focused on.

\section{Acknowledgements}

The study was supported by the Zhejiang provincial health bureau foundation (no. 2009 A125) and the National Natural Science Foundation of China (no. 30901327).

\section{References}

1. Babay HA, Twum-Danso K, Kambal AM and Al-Otaibi FE: Bloodstream infections in pediatric patients. Saudi Med J 26: $1555-1561,2005$

2. Lehrnbecher T, Varwig D, Kaiser J, Reinhardt D, Klingebiel T and Creutzig U: Infectious complications in pediatric acute myeloid leukemia: analysis of the prospective multi-institutional clinical trial AML-BFM 93. Leukemia 18: 72-77, 2004.

3. Bononi A, Lanza F, Ferrari L, et al: Predictive value of hematological and phenotypical parameters on postchemotherapy leukocyte recovery. Cytometry B Clin Cytom 76: 328-333, 2009.

4. Tromp YH, Daenen SM, Sluiter WJ and Vellenga E: The predictive value of interleukin-8 (IL-8) in hospitalised patients with fever and chemotherapy-induced neutropenia. Eur J Cancer 45: 596-600, 2009.

5. Yang Y and Shen X: Emphasis on study of infectious diseases in China. Zhonghua Yi Xue Za Zhi 81: 899-900, 2001 (In Chinese).

6. Tang Y, Liao C, Xu X, et al: Evaluation of Th1/Th2 cytokines as a rapid diagnostic tool for severe infection in paediatric haematology/oncology patients by the use of cytometric bead array technology. Clin Microbiol Infect 17: 1666-1673, 2011.

7. Robinson JO, Lamoth F, Bally F, Knaup M, Calandra T and Marchetti O: Monitoring procalcitonin in febrile neutropenia: what is its utility for initial diagnosis of infection and reassessment in persistent fever? PLoS One 6: e18886, 2011.

8. Dowzicky MJ and Park CH: Update on antimicrobial susceptibility rates among Gram-negative and Gram-positive organisms in the United States: results from the Tigecycline Evaluation and Surveillance Trial (TEST) 2005 to 2007. Clin Ther 30: 2040-2050, 2008.

9. Abdul Rasool Hassan B, Yusoff ZB and Bin Othman S: Association of neutropenia onset and severity with chemotherapy regimens and schedules. Asian Pac J Cancer Prev 12: 1425-1428, 2011.

10. Meric M, Willke A, Caglayan C and Toker K: Intensive care unit-acquired infections: incidence, risk factors and associated mortality in a Turkish university hospital. Jpn J Infect Dis 58: 297-302, 2005.

11. Prowle JR, Echeverri JE, Ligabo EV, et al: Acquired bloodstream infection in the intensive care unit: incidence and attributable mortality. Crit Care 15: R100, 2011.

12. Lyytikäinen O, Lumio J, Sarkkinen H, Kolho E, Kostiala A and Ruutu P; Hospital Infection Surveillance Team: Nosocomial bloodstream infections in Finnish hospitals during 1999-2000. Clin Infect Dis 35: e14-e19, 2002.

13. Velasco E, Byington R, Martins CS, Schirmer M, Dias LC and Gonçalves VM: Bloodstream infection surveillance in a cancer centre: a prospective look at clinical microbiology aspects. Clin Microbiol Infect 10: 542-549, 2004.

14. Celkan T, Ozkan A, Apak H, et al: Bacteremia in childhood cancer. J Trop Pediatr 48: 373-377, 2002.

15. Hufnagel M, Burger A, Bartelt S, Henneke P and Berner R: Secular trends in pediatric bloodstream infections over a 20-year period at a tertiary care hospital in Germany. Eur J Pediatr 167: 1149-1159, 2008

16. Hughes WT, Armstrong D, Bodey GP, et al: 2002 guidelines for the use of antimicrobial agents in neutropenic patients with cancer. Clin Infect Dis 34: 730-751, 2002.

17. Hughes WT, Armstrong D, Bodey GP, et al; Infectious Diseases Society of America: 1997 guidelines for the use of antimicrobial agents in neutropenic patients with unexplained fever. Clin Infect Dis 25: 551-573, 1997.

18. Vento $\mathrm{S}$ and Cainelli F: Infections in patients with cancer undergoing chemotherapy: aetiology, prevention, and treatment. Lancet Oncol 4: 595-604, 2003.

19. Mor M, Gilad G, Kornreich L, Fisher S, Yaniv I and Levy I: Invasive fungal infections in pediatric oncology. Pediatr Blood Cancer 56: 1092-1097, 2011. 